\title{
Indirect Inverse Substructuring Method for Multibody Product Transport System with Rigid and Flexible Coupling
}

\author{
Jun Wang, ${ }^{1,2,3}$ Li-xin Lu, ${ }^{1,3}$ Pengjiang Qian, ${ }^{4}$ Li-qiang Huang, ${ }^{2}$ \\ Yan Hua, ${ }^{1,3}$ and Guang-yi $\mathrm{Pu}^{3}$ \\ ${ }^{1}$ Department of Packaging Engineering, Jiangnan University, Wuxi 214122, China \\ ${ }^{2}$ Tianjin Key Laboratory of Pulp \& Paper, Tianjin University of Science \& Technology, Tianjin 300457, China \\ ${ }^{3}$ Jiangsu Key Laboratory of Advanced Food Manufacturing Equipment and Technology, Jiangnan University, Wuxi 214122, China \\ ${ }^{4}$ School of Digital Media, Jiangnan University, Wuxi 214122, China
}

Correspondence should be addressed to Jun Wang; wangj_1982@jiangnan.edu.cn and Li-xin Lu; lulx@jiangnan.edu.cn

Received 16 July 2014; Accepted 30 October 2014

Academic Editor: De Gao

Copyright (c) 2015 Jun Wang et al. This is an open access article distributed under the Creative Commons Attribution License, which permits unrestricted use, distribution, and reproduction in any medium, provided the original work is properly cited.

\begin{abstract}
The aim of this paper is to develop a new frequency response function- (FRF-) based indirect inverse substructuring method without measuring system-level FRFs in the coupling DOFs for the analysis of the dynamic characteristics of a three-substructure coupled product transport system with rigid and flexible coupling. By enforcing the dynamic equilibrium conditions at the coupling coordinates and the displacement compatibility conditions, a closed-form analytical solution to inverse substructuring analysis of multisubstructure coupled product transport system is derived based on the relationship of easy-to-monitor component-level FRFs and the system-level FRFs at the coupling coordinates. The proposed method is validated by a lumped mass-spring-damper model, and the predicted coupling dynamic stiffness is compared with the direct computation, showing exact agreement. The method developed offers an approach to predict the unknown coupling dynamic stiffness from measured FRFs purely. The suggested method may help to obtain the main controlling factors and contributions from the various structure-borne paths for product transport system.
\end{abstract}

\section{Introduction}

Packaging dynamics for protection of the packaged product has attracted increasingly more attention during the last two decades [1-9]. In distribution process, the product, packaging, and vehicle constitute a complex built-up product transport system. The packaged product will be damaged when the dynamic response due to the environmental vibration and shock exceeds some limit value [10]. To protect the packaged product, one needs to isolate the packaged item with flexible cushions. Hence, it is of significant importance to identify the coupling dynamic stiffness between packaged product and vehicle system for cushioning packaging design $[11,12]$. However, the interaction between the packaged product and vehicle is extremely complicated, including internal and external cushion, container, pallet, nail, and rope. It is difficult to predict the coupling dynamic stiffness accurately by reported identification methods [12-14], as some of the frequency response functions (FRFs) required cannot be easily measured between the complex coupling interfaces for a product transport system. To address this issue, Zhen et al. [15] proposed an inverse formulation that the FRFs of individual components and dynamic characteristics of the coupling elements can be predicted directly from the system-level FRFs. Although this technique includes several mathematical operations that might be very sensitive to measurement errors and/or and inconsistencies, it has its unique advantages in various application fields since no componentlevel spectra response is needed. Then it was applied to study the dynamics of product transport system [16, 17], showing its great application prospect in industry. In spite of those promising successful applications, the two-component structure assumption of the inverse substructure theory proposed by Zhen restricts its application for a complex product transport system. The discretely connected system should be taken as a multisubstructure coupled system in order to perform 


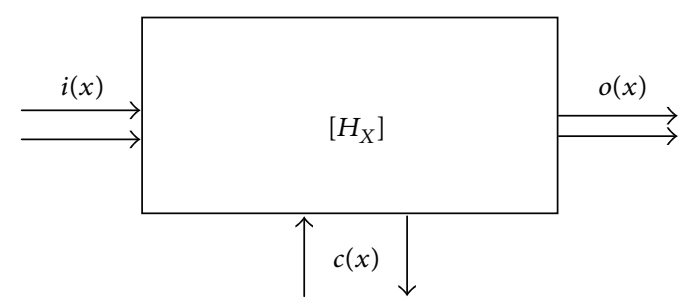

FIGURE 1: A general substructure representation.

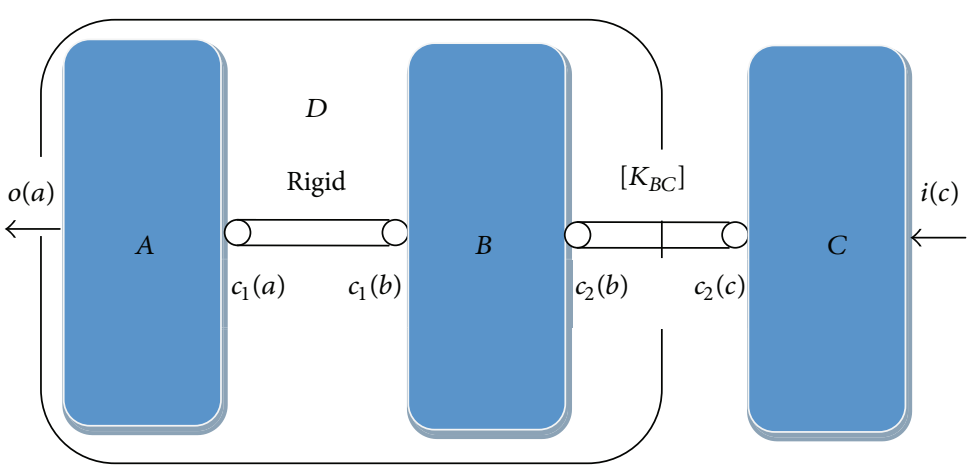

FIGURE 2: Single-coordinate coupled three-substructure system.

the parametric analysis of each substructure and connector more efficiently. In our recent published work $[18,19]$, the inverse substructuring method of three-component coupled product transport system for both single coordinate coupling case and multicoordinate coupling case was proposed and applied to perform the parameter analysis of product transport system. However, in the previous work, the dynamic stiffness of the coupling interface was predicted purely from the system-level FRFs. Actually, the system-level FRFs from coupling degree of freedoms (DOFs) may not be measured accurately due to the difficulties of vibration excitation and measurement for the coupled interface between packaged product and vehicle within the limited space. In this paper, an indirect inverse substructuring method is proposed to identify the coupling dynamic stiffness. Instead of identification of the coupling dynamic stiffness only from system-level FRFs, the proposed method aims to obtain the packaging stiffness accurately from easy-to-monitor DOFs, which will avoid the difficulties of vibration excitation and measurement due to space limitation. The accuracy of the proposed theory is first validated by a lumped parameter model.

\section{Indirect Inverse Substructuring Method for Multicomponent Coupled Product Transport System with Rigid and Flexible Coupling}

A free substructure $X$ is shown in Figure 1, where $o(x)$, $i(x)$, and $c(x)$ are the response, excitation, and coupling coordinates, respectively. At the coupling coordinate, the substructure is connected to the rest of the system. The governing equations of motion can be written as [16]

$$
\left[M_{X}\right]\left\{\ddot{Y}_{X}\right\}+\left[C_{X}\right]\left\{\dot{Y}_{X}\right\}+\left[K_{X}\right]\left\{Y_{X}\right\}=\left\{F_{X}\right\},
$$

where $\left[M_{X}\right],\left[C_{X}\right]$, and $\left[K_{X}\right]$ are the mass, damping, and stiffness matrices, respectively, and the subscript $X$ refers to substructure $X(X=A, B, C, S)$. Assuming forced periodic excitation, the steady-state vibratory response in frequency domain can be expressed as

$$
\begin{aligned}
{\left[H_{X}\right]=\left[\begin{array}{ll}
{\left[H_{X}\right]_{o(x) i(x)}} & {\left[H_{X}\right]_{o(x) c(x)}} \\
{\left[H_{X}\right]_{c(x) i(x)}} & {\left[H_{X}\right]_{c(x) c(x)}}
\end{array}\right] } \\
=-\omega^{2}\left(-\omega^{2}\left[M_{X}\right]+j \omega\left[C_{X}\right]+\left[K_{X}\right]\right)^{-1}, \\
X=A, B, C, S,
\end{aligned}
$$

where $\omega=2 \pi f$ is the excitation frequency. This form is used to generate the necessary frequency response function (FRF) matrix of the substructure $X$. Here, $H_{X, g k}$ represents the frequency response function matrices of substructure $X$ (response at $g$ coordinate due to an excitation at $k$ coordinate); for example, for $X=A$ and $x=a, H_{A, o(a) i(a)}$ is the transfer response function between the excitation coordinate $i(a)$ and response coordinate $o(a)$ of substructure A.

Consider a system with three substructures $A, B$, and $C$ coupled at interface $c_{1}$ by a rigid connector between $A$ and $B$ and at interface $c_{2}$ by a flexible connector defined as $K_{B C}$ between $B$ and $C$ (see Figure 2).

By applying the substructuring method, the system-level FRFs can be predicted from the component-level FRFs. 


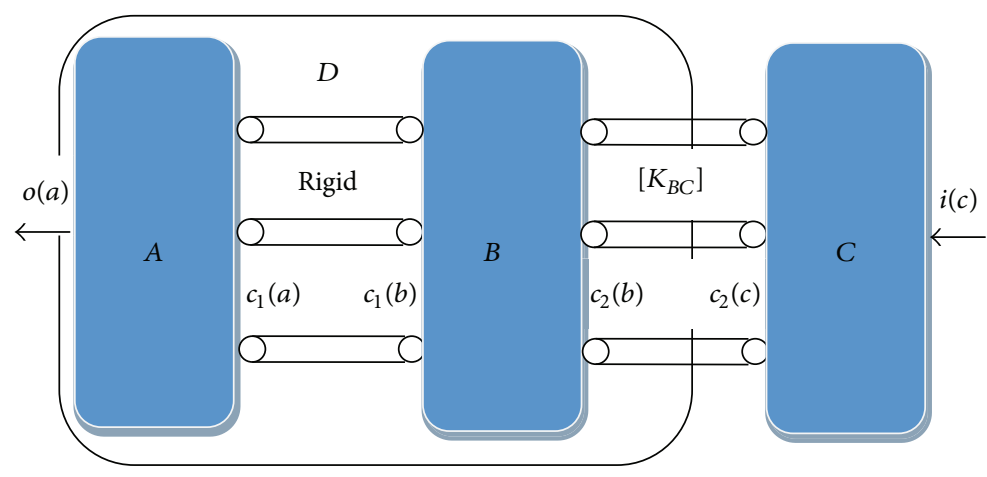

FIgURE 3: Multicoordinate coupled three-substructure system.

First, take the structure $D$ coupled by substructure $A$ and substructure $B$ as a system, and the FRFs of structure $D$ can be expressed by the FRFs of substructure $A$ and substructure $B$ as $[18,19]$

$$
\begin{aligned}
& {\left[\begin{array}{ccc}
H_{D, o(a) i(a)} & H_{D, o(a) c_{1}(x)} & H_{D, o(a) i(b)} \\
H_{D, c_{1}(x) i(a)} & H_{D, c_{1}(x) c_{1}(x)} & H_{D, c_{1}(x) i(b)} \\
H_{D, o(b) i(a)} & H_{D, o(b) c_{1}(x)} & H_{D, o(b) i(b)}
\end{array}\right]} \\
& =\left[\begin{array}{ccc}
H_{A, o(a) i(a)} & H_{A, o(a) c_{1}(x)} & 0 \\
H_{X, c_{1}(x) i(a)} & H_{X, c_{1}(x) c_{1}(x)} & H_{X, c_{1}(x) i(b)} \\
0 & H_{B, o(b) c_{1}(x)} & H_{B, o(b) i(b)}
\end{array}\right] \\
& -\left[\begin{array}{c}
\alpha H_{A, o(a) c_{1}(a)} \\
H_{X, c_{1}(x) c_{1}(x)} \\
\beta H_{B, o(b) c_{1}(b)}
\end{array}\right]\left[D_{A B}\right] \\
& \times\left[\begin{array}{lll}
\alpha H_{A, c_{1}(a) i(a)} & H_{X, c_{1}(x) c_{1}(x)} & \beta H_{B, c_{1}(b) i(b)}
\end{array}\right],
\end{aligned}
$$

where

$$
\begin{aligned}
& \alpha=\left\{\begin{array}{lll}
1 & x=a, & X=A \\
-1 & x=b, & X=B,
\end{array}\right. \\
& \beta= \begin{cases}1 & x=b, \quad X=B \\
-1 & x=a, \quad X=A,\end{cases}
\end{aligned}
$$

and $D_{A B}=\left(H_{A, c_{1}(a) c_{1}(a)}+H_{B, c_{1}(b) c_{1}(b)}\right)^{-1}$.

Then, take the system $S$ as a coupled one by substructure $D$ and substructure $C$ (here, structure $D$ is treated as a component), and the FRFs of the system $S$ can be expressed by the FRFs of substructure $D$ and substructure $C$ as

$$
\begin{gathered}
{\left[\begin{array}{ccc}
H_{S, o(d) i(d)} & H_{S, o(d) c_{2}(x)} & H_{S, o(d) i(c)} \\
H_{S, c_{2}(x) i(d)} & H_{S, c_{2}(x) c_{2}(x)} & H_{S, c_{2}(x) i(c)} \\
H_{S, o(c) i(d)} & H_{S, o(c) c_{2}(x)} & H_{S, o(c) i(c)}
\end{array}\right]} \\
=\left[\begin{array}{ccc}
H_{D, o(d) i(d)} & H_{D, o(d) c_{2}(x)} & 0 \\
H_{X, c_{2}(x) i(d)} & H_{X, c_{2}(x) c_{2}(x)} & H_{X, c_{2}(x) i(c)} \\
0 & H_{C, o(c) c_{2}(x)} & H_{C, o(c) i(c)}
\end{array}\right]
\end{gathered}
$$

$$
\begin{aligned}
& -\left[\begin{array}{c}
\alpha H_{D, o(d) c_{2}(d)} \\
H_{X, c_{2}(x) c_{2}(x)} \\
\beta H_{C, o(c) c_{2}(c)}
\end{array}\right]\left[D_{D C}\right] \\
& \times\left[\begin{array}{lll}
\alpha H_{D, c_{2}(d) i(d)} & H_{X, c_{2}(x) c_{2}(x)} & \beta H_{C, c_{2}(c) i(c)}
\end{array}\right],
\end{aligned}
$$

where

$$
\begin{aligned}
& \alpha= \begin{cases}1 & x=d, X=D \\
-1 & x=c,\end{cases} \\
& \beta= \begin{cases}1 & x=c, X=C \\
-1 & x=d, \quad X=D,\end{cases}
\end{aligned}
$$

and $D_{D C}=\left(H_{D, c_{2}(b) c_{2}(b)}+H_{C, c_{2}(c) c_{2}(c)}-\omega^{2} K_{B C}{ }^{-1}\right)^{-1}$.

From (5) and (3), it can be seen that $H_{S}$ is expressed by $H_{D}$ and $H_{C}$, and $H_{D}$ is expressed by $H_{A}$ and $H_{B}$. Substituting (5) into (3) yields the FRFs of the system $S$ expressed by the FRFs of the three substructures, $A, B$, and $C$. Because the response at $o(a)$ coordinate of substructure $A$ due to an excitation at $i(c)$ coordinate of substructure $C$ is most concerned, we give here the explicit expression of the system-level FRF $H_{s, o(a) i(c)}$ by the FRFs of the three substructures $[18,19]$ :

$$
\begin{aligned}
& H_{S, o(a) i(c)} \\
& =H_{A, o(a) c_{1}(a)}\left\{H_{A, c_{1}(a) c_{1}(a)}+H_{B, c_{1}(b) c_{1}(b)}\right\}^{-1} \\
& \times \\
& \quad H_{B, c_{1}(b) c_{2}(b)} \\
& \times\left\{H_{B, c_{2}(b) c_{2}(b)}-H_{B, c_{2}(b) c_{1}(b)}\right. \\
& \quad \times\left(H_{A, c_{1}(a) c_{1}(a)}+H_{B, c_{1}(b) c_{1}(b)}\right)^{-1} H_{B, c_{1}(b) c_{2}(b)} \\
& \left.\quad+H_{C, c_{2}(c) c_{2}(c)}-\omega^{2} K_{B C}^{-1}\right\}^{-1} H_{C, c_{2}(c) i(c)} .
\end{aligned}
$$

Similarly, one can obtain the system-level FRF for multicoordinate coupling case as (see Figure 3)

$$
\begin{aligned}
& {\left[H_{S}\right]_{o(a) i(c)}} \\
& \quad=\left[H_{A}\right]_{o(a) c_{1}(a)}
\end{aligned}
$$




$$
\begin{aligned}
& \times\left\{\left[H_{A}\right]_{c_{1}(a) c_{1}(a)}+\left[H_{B}\right]_{\mathcal{c}_{1}(b) c_{1}(b)}\right\}^{-1}\left[H_{B}\right]_{c_{1}(b) c_{2}(b)} \\
& \times\left\{\left[H_{B}\right]_{\mathcal{c}_{2}(b) c_{2}(b)}-\left[H_{B}\right]_{\mathcal{c}_{2}(b) c_{1}(b)}\right. \\
& \times\left(\left[H_{A}\right]_{\mathcal{c}_{1}(a) c_{1}(a)}+\left[H_{B}\right]_{c_{1}(b) c_{1}(b)}\right)^{-1}\left[H_{B}\right]_{c_{1}(b) c_{2}(b)} \\
& \left.\quad+\left[H_{C}\right]_{\mathcal{C}_{2}(c) c_{2}(c)}-\omega^{2}\left[K_{B C}\right]^{-1}\right\}^{-1}\left[H_{C}\right]_{\mathcal{c}_{2}(c) i(c)} .
\end{aligned}
$$

The above derivation develops a method for a threesubstructure coupled system to obtain the system-level responses from the prior knowledge of FRFs of the three substructures and the dynamic stiffness of the coupling interfaces. However, for many complex structure systems, the substructure-level FRFs may not be easily obtained. Besides, the dynamic stiffness of the coupling interface may be unknown. If the physical system exists but is not conveniently separable into two or more substructures, it is desirable to express the problem in terms of measurable system-level FRFs.

2.1. Closed-Form Solution for Single Coupling. The coupled system is first decoupled as two substructures, $D$ and $C$, as illustrated in Figure 2. The force compatibility of the coupling coordinates between substructure $D$ and substructure $C$ leads to the following equation:

$$
\begin{aligned}
& \left\{\begin{array}{l}
F_{c_{2}(d)} \\
F_{c_{2}(c)}
\end{array}\right\} \\
& =\left[\begin{array}{cc}
H_{D, c_{2}(d) c_{c}(d)}^{-1}-\omega^{-2} K_{B C} & \omega^{-2} K_{B C} \\
\omega^{-2} K_{B C} & H_{C, c_{2}(c) c_{2}(c)}^{-1}-\omega^{-2} K_{B C}
\end{array}\right]\left\{\begin{array}{c}
\ddot{Y}_{c_{2}(d)} \\
\ddot{Y}_{c_{2}(c)}
\end{array}\right\} .
\end{aligned}
$$

The response vector of the coupling coordinates can then be solved as

$$
\begin{aligned}
& \left\{\begin{array}{c}
\ddot{Y}_{c_{2}(d)} \\
\ddot{Y}_{c_{2}(c)}
\end{array}\right\} \\
& =[\Gamma]^{-1}\left[\begin{array}{cc}
H_{C, c_{2}(c) c_{2}(c)}^{-1}-\omega^{-2} K_{B C} & -\omega^{-2} K_{B C} \\
-\omega^{-2} K_{B C} & H_{D, c_{2}(d) c_{2}(d)}^{-1}-\omega^{-2} K_{B C}
\end{array}\right] \\
& \quad \times\left\{\begin{array}{l}
F_{c_{2}(d)} \\
F_{c_{2}(c)}
\end{array}\right\},
\end{aligned}
$$

where

$$
[\Gamma]=\left|\begin{array}{cc}
H_{D, c_{2}(d) c_{2}(d)}^{-1}-\omega^{-2} K_{B C} & \omega^{-2} K_{B C} \\
\omega^{-2} K_{B C} & H_{C, c_{2}(c) c_{2}(c)}^{-1}-\omega^{-2} K_{B C}
\end{array}\right| .
$$

The coefficient matrix in (10) is the transfer matrix $H_{S}$ between the coupling coordinates on substructure $D$ and substructure $C$, and it can be written in terms of system-level FRFs as

$$
\left\{\begin{array}{l}
\ddot{Y}_{c_{c_{2}}(d)} \\
\ddot{Y}_{c_{2}(c)}
\end{array}\right\}=\left[\begin{array}{ll}
H_{S, c_{2}(d) c_{2}(d)} & H_{S, c_{2}(d) c_{2}(c)} \\
H_{S, c_{2}(c) c_{2}(d)} & H_{S, c_{2}(c) c_{2}(c)}
\end{array}\right]\left\{\begin{array}{c}
F_{c_{2}(d)} \\
F_{c_{2}(c)}
\end{array}\right\} .
$$

Comparing (10) and (12) and recalling (5) reveal a series of equations relating the component-level FRFs to systemlevel FRFs, from which we can obtain the expression of the coupling dynamic stiffness as depicted in

$$
K_{B C}=\frac{-\omega^{2} H_{S, c_{2}(b) c_{2}(c)}}{H_{S, c_{2}(b) c_{2}(b)} H_{S, c_{2}(c) c_{2}(c)}-H_{S, c_{2}(b) c_{2}(c)}^{2}} .
$$

Equation (13) provides an inverse method to predict the coupling dynamic stiffness for product transport system. But the problem remaining is that some system-level FRFs cannot be easily measured from the coupling DOFs such as $H_{s, c_{2}(c) c_{2}(c)}$. To overcome this shortcoming, we recall back (7), make an inverse formulation, and then get

$$
\begin{aligned}
K_{B C}=-\omega^{2}\{ & H_{C, c_{2}(c) i(c)} H_{S, o(a) i(c)}^{-1} H_{A, o(a) c_{1}(a)} \\
& \times\left[H_{A, c_{1}(a) c_{1}(a)}+H_{B, c_{1}(b) c_{1}(b)}\right]^{-1} H_{B, c_{1}(b) c_{2}(b)} \\
& -H_{B, c_{2}(b) c_{2}(b)}+H_{B, c_{2}(b) c_{1}(b)} \\
& \times\left[H_{A, c_{1}(a) c_{1}(a)}+H_{B, c_{1}(b) c_{1}(b)}\right]^{-1} \\
& \left.\times H_{B, c_{1}(b) c_{2}(b)}-H_{C, c_{2}(c) c_{2}(c)}\right\}^{-1}
\end{aligned}
$$

From (14), the coupling dynamic stiffness of the interface between packaged product and vehicle can be predicted from both system-level FRFs and component-level FRFs; all of the FRFs from DOFs of coupling $c_{2}$ are measured in component level, which avoids the difficulties of vibration excitation and pickup. As the coupling dynamic stiffness is predicted from both system-level FRFs and component-level FRFs, we call the method proposed indirect inverse substructuring method.

2.2. Closed-Form Solution for Multicoordinate Coupling. As depicted in Figure 3, the components are connected by a set of $\left[K_{C}\right]$ of dimension $p>1$. In many cases, $\left[K_{C}\right]$ is nearly diagonal especially in product transport system. Similar to the single coupling problem, the dynamic equilibrium conditions are applied to the coupling coordinates on both sides of the connecting springs to obtain

$$
\begin{aligned}
& \left\{\begin{array}{l}
\left\{F_{c_{2}(d)}\right\} \\
\left\{F_{c_{2}(c)}\right\}
\end{array}\right\} \\
& =\left[\begin{array}{cc}
{\left[H_{D}\right]_{\mathcal{C}_{2}(d) c_{2}(d)}^{-1}-\omega^{-2}\left[K_{B C}\right]} & \omega^{-2}\left[K_{B C}\right] \\
\omega^{-2}\left[K_{B C}\right] & {\left[H_{C}\right]_{\mathcal{c}_{2}(c) \mathcal{c}_{2}(c)}^{-1}-\omega^{-2}\left[K_{B C}\right]}
\end{array}\right] \\
& \times\left\{\left\{\begin{array}{l}
\left.\ddot{Y}_{c_{2}(d)}\right\} \\
\left.\ddot{Y}_{c_{2}(c)}\right\}
\end{array}\right\}\right.
\end{aligned}
$$

which is inverted to express the response vector of the coupling coordinates as 


$$
\begin{aligned}
\left\{\begin{array}{l}
\left\{\ddot{Y}_{\mathcal{c}_{2}(d)}\right\} \\
\left\{\ddot{Y}_{c_{2}(c)}\right\}
\end{array}\right\}= & {\left[\begin{array}{c}
{[\Gamma]^{-1}\left([I]-\omega^{2}\left[H_{C}\right]_{\mathcal{c}_{2}(c) c_{2}(c)}^{-1}\left[K_{B C}\right]^{-1}\right)} \\
{[\Gamma]^{-T}}
\end{array} \quad \begin{array}{c}
{[\Gamma]^{-1}} \\
\left(-\omega^{2}\left[K_{B C}\right]^{-1}\left[H_{D}\right]_{c_{2}(d) c_{2}(d)}^{-1}+[I]\right)[\Gamma]^{-1}
\end{array}\right] } \\
& \times\left\{\begin{array}{c}
\left\{F_{c_{2}(d)}\right\} \\
\left\{F_{c_{2}(c)}\right\}
\end{array}\right\},
\end{aligned}
$$

where $[\Gamma]=-\omega^{2}\left[H_{C}\right]_{\mathcal{C}_{2}(c) \mathcal{c}_{2}(c)}^{-1}\left[K_{B C}\right]^{-1}\left[H_{D}\right]_{\mathcal{C}_{2}(d) \mathcal{c}_{2}(d)}^{-1}+$ $\left[H_{C}\right]_{\mathcal{C}_{2}(c) \mathcal{c}_{2}(c)}^{-1}+\left[H_{D}\right]_{\mathcal{C}_{2}(d) \mathfrak{c}_{\mathcal{C}(d)}}^{-1}$.

The coefficient matrix in (16) is the transfer matrix $\left[H_{S}\right]$ between the coupling coordinates on substructure $D$ and substructure $C$, and it can be written in terms of system-level FRFs as

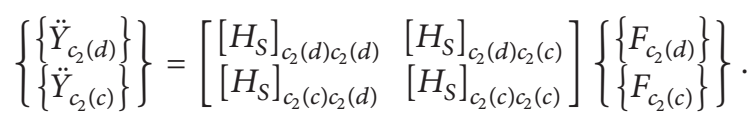

Comparing (16) and (17) and recalling (8) reveal a series of equations relating the component-level FRFs to systemlevel FRFs, from which we can obtain the expression of the coupling dynamic stiffness between $B$ and $C$ as in (18) after a lengthy derivation:

$$
\begin{aligned}
& {\left[K_{B C}\right]} \\
& \quad=-\omega^{2} \\
& \quad \times\left(\left[H_{S}\right]_{\mathcal{C}_{2}(b) \mathcal{C}_{2}(b)}\left[H_{S}\right]_{\mathcal{C}_{2}(b) \mathcal{C}_{2}(c)}^{-T}\left[H_{S}\right]_{\mathcal{C}_{2}(c) \mathcal{c}_{2}(c)}-\left[H_{S}\right]_{\mathcal{C}_{2}(b) \mathcal{C}_{2}(c)}\right)^{-1} .
\end{aligned}
$$

Equation (18) provides an inverse method to predict the coupling dynamic stiffness for product transport system. But the remaining problem is that some system-level FRFs cannot be easily measured from the coupling DOFs such as $\left[H_{s}\right]_{\mathcal{C}_{2}(c) \mathcal{c}_{2}(c)}$. To overcome this shortcoming, we recall back (8) and make an inverse formulation:

$$
\begin{aligned}
& {\left[K_{B C}\right]=-\omega^{2}} \\
& \times\left\{\left[H_{C}\right]_{\mathcal{C}_{2}(c) i(c)}\left[H_{S}\right]_{o(a) i(c)}^{-1}\left[H_{A}\right]_{o(a) c_{1}(a)}\right. \\
& \times\left(\left[H_{A}\right]_{\mathcal{c}_{1}(a) \mathfrak{c}_{1}(a)}+\left[H_{B}\right]_{\mathcal{c}_{1}(b) c_{1}(b)}\right)^{-1} \\
& \times\left[H_{B}\right]_{\mathcal{1}_{1}(b) \mathfrak{c}_{2}(b)}-\left[H_{B}\right]_{\mathcal{c}_{2}(b) \mathfrak{c}_{2}(b)}+\left[H_{B}\right]_{\mathcal{c}_{2}(b) \mathfrak{c}_{1}(b)} \\
& \times\left(\left[H_{A}\right]_{\mathcal{c}_{1}(a) \mathfrak{c}_{1}(a)}+\left[H_{B}\right]_{\mathcal{C}_{1}(b) \mathcal{c}_{1}(b)}\right)^{-1} \\
& \left.\times\left[H_{B}\right]_{\mathcal{c}_{1}(b) c_{2}(b)}-\left[H_{C}\right]_{\mathcal{c}_{2}(c) c_{2}(c)}\right\}^{-1},
\end{aligned}
$$

where $[\Psi]=\left[H_{A}\right]_{o(a) c_{1}(a)}\left(\left[H_{A}\right]_{\mathcal{C}_{1}(a) c_{1}(a)}+\right.$ $\left.\left[H_{B}\right]_{\mathcal{c}_{1}(b) \mathfrak{c}_{1}(b)}\right)^{-1}\left[H_{B}\right]_{\mathcal{c}_{1}(b) \mathfrak{c}_{\mathcal{G}}(b)}$.

Equation (19) can be used to predict the coupling dynamic stiffness from both system-level FRFs and component-level FRFs, avoiding the difficulties of vibration excitation and pickup.

\section{Numerical Validation Using a Lumped Parameter Model}

To verify the indirect inverse substructure proposed above, a lumped mass-spring-damper model with three substructures, shown in Figure 4, is taken as an example. The specific parameters of the system are listed in Table 1 . The necessary FRFs of the free substructures and the coupled system are generated from (2), and the computed system response functions as well as the necessary component response functions are used to predict the coupling dynamic stiffness applying (19) for packaging interface. Then, the results are compared to direct calculations, as shown in Figure 5. Specifically, for $H_{S, i j}, i=(1),(2), j=(7),(8)$, and $H_{A, i j}, i, j=(2),(3)$, and $i=(1),(2), j=(2),(3), H_{B, i j}, i, j=(1),(2)$, and $i, j=(3),(4)$, and $i=(1),(2), j=(3),(4)$, and $H_{C, i j}, i=(6),(7), j=(7),(8)$ and $i, j=(6),(7)$. All necessary FRFs are calculated from (2). The matrices for components $A, B, C$, and $S$ are expressed explicitly as follows:

component $A$ :

$$
\begin{aligned}
& {\left[M_{A}\right]=\left[\begin{array}{ccc}
m_{1} & 0 & 0 \\
0 & m_{21} & 0 \\
0 & 0 & m_{22}
\end{array}\right],} \\
& {\left[K_{A}\right]=\left[\begin{array}{ccc}
k_{11}+k_{12} & -k_{11} & -k_{12} \\
-k_{11} & k_{11} & 0 \\
-k_{12} & 0 & k_{12}
\end{array}\right],} \\
& {\left[C_{A}\right]=\left[\begin{array}{ccc}
c_{11}+c_{12} & -c_{11} & -c_{12} \\
-c_{11} & c_{11} & 0 \\
-c_{12} & 0 & c_{12}
\end{array}\right] ;}
\end{aligned}
$$

component $B$ :

$$
\begin{aligned}
& {\left[M_{B}\right]=\left[\begin{array}{cccc}
m_{3} & 0 & 0 & 0 \\
0 & m_{4} & 0 & 0 \\
0 & 0 & m_{5} & 0 \\
0 & 0 & 0 & m_{6}
\end{array}\right],} \\
& {\left[K_{B}\right]=\left[\begin{array}{cccc}
k_{2}+k_{3} & -k_{2} & -k_{3} & 0 \\
-k_{2} & k_{2}+k_{4} & 0 & -k_{4} \\
-k_{3} & 0 & k_{3}+k_{5} & -k_{5} \\
0 & -k_{4} & -k_{5} & k_{4}+k_{5}
\end{array}\right],} \\
& {\left[C_{B}\right]=\left[\begin{array}{cccc}
c_{2}+c_{3} & -c_{2} & -c_{3} & 0 \\
-c_{2} & c_{2}+c_{4} & 0 & -c_{4} \\
-c_{3} & 0 & c_{3}+c_{5} & -c_{5} \\
0 & -c_{4} & -c_{5} & c_{4}+c_{5}
\end{array}\right] ;}
\end{aligned}
$$


TABLE 1: Model parameters for lumped parameter model shown in Figure 4.

\begin{tabular}{lcclcccccccc}
\hline & \multicolumn{3}{c}{ Mass $(\mathrm{kg})$} & \multicolumn{3}{c}{ Stiffness (N/m) } & \multicolumn{3}{c}{ Damping (Ns/m) } \\
\hline$m_{1}$ & $m_{21}$ & $m_{22}$ & $m_{3}$ & $k_{11}$ & $k_{12}$ & $k_{2}$ & $k_{3}$ & $c_{11}$ & $c_{12}$ & $c_{2}$ & $c_{3}$ \\
20 & 8 & 8 & 22 & 7200 & 7200 & 3600 & 4200 & 15 & 15 & 12 & 15 \\
$m_{4}$ & $m_{5}$ & $m_{6}$ & $m_{7}$ & $k_{4}$ & $k_{5}$ & $k_{6}$ & $k_{7}$ & $c_{4}$ & $c_{5}$ & $c_{6}$ & $c_{7}$ \\
18 & 15 & 12 & 16 & 7900 & 6800 & 6000 & 5800 & 10 & 8 & 22 & 8 \\
$m_{8}$ & $m_{9}$ & $/$ & $/$ & $k_{57}$ & $k_{68}$ & $/$ & $/$ & $c_{57}$ & $c_{68}$ & $/$ & $/$ \\
16 & 40 & $/$ & $/$ & 5800 & 6200 & $/$ & $/$ & 16 & 12 & $/$ \\
\hline
\end{tabular}

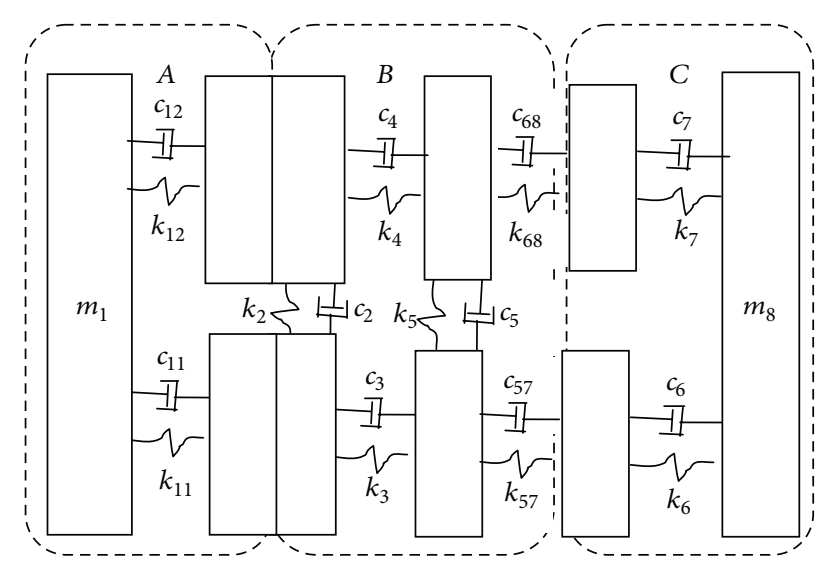

FIGURE 4: A lumped mass-spring-damper model. component $C$ :

$$
\begin{aligned}
& {\left[M_{C}\right]=\left[\begin{array}{ccc}
m_{7} & 0 & 0 \\
0 & m_{8} & 0 \\
0 & 0 & m_{9}
\end{array}\right],} \\
& {\left[K_{C}\right]=\left[\begin{array}{ccc}
k_{6} & 0 & -k_{6} \\
0 & k_{7} & -k_{7} \\
-k_{6} & -k_{7} & k_{6}+k_{7}
\end{array}\right],} \\
& {\left[C_{C}\right]=\left[\begin{array}{ccc}
c_{6} & 0 & -c_{6} \\
0 & c_{7} & -c_{7} \\
-c_{6} & -c_{7} & c_{6}+c_{7}
\end{array}\right] ;}
\end{aligned}
$$

$$
\begin{aligned}
& {\left[M_{S}\right]=\left[\begin{array}{cccccccc}
m_{1} & 0 & 0 & 0 & 0 & 0 & 0 & 0 \\
0 & m_{21}+m_{3} & 0 & 0 & 0 & 0 & 0 & 0 \\
0 & 0 & m_{22}+m_{4} & 0 & 0 & 0 & 0 & 0 \\
0 & 0 & 0 & m_{5} & 0 & 0 & 0 & 0 \\
0 & 0 & 0 & 0 & m_{6} & 0 & 0 & 0 \\
0 & 0 & 0 & 0 & 0 & m_{7} & 0 & 0 \\
0 & 0 & 0 & 0 & 0 & 0 & m_{8} & 0 \\
0 & 0 & 0 & 0 & 0 & 0 & 0 & m_{9}
\end{array}\right]} \\
& {\left[K_{S}\right]=\left[\begin{array}{cccccccc}
k_{11}+k_{12} & -k_{11} & -k_{12} & 0 & 0 & 0 & 0 & 0 \\
-k_{11} & k_{11}+k_{2}+k_{3} & -k_{2} & -k_{3} & 0 & 0 & 0 & 0 \\
-k_{12} & -k_{2} & k_{12}+k_{2}+k_{4} & 0 & -k_{4} & 0 & 0 & 0 \\
0 & -k_{3} & 0 & k_{3}+k_{5}+k_{57} & -k_{5} & -k_{57} & 0 & 0 \\
0 & 0 & -k_{4} & -k_{5} & k_{4}+k_{5}+k_{68} & 0 & -k_{68} & 0 \\
0 & 0 & 0 & -k_{57} & 0 & k_{6}+k_{57} & 0 & -k_{6} \\
0 & 0 & 0 & 0 & -k_{68} & 0 & k_{7}+k_{68} & -k_{7} \\
0 & 0 & 0 & 0 & 0 & -k_{6} & -k_{7} & k_{6}+k_{7}
\end{array}\right]} \\
& {\left[C_{S}\right]=\left[\begin{array}{cccccccc}
c_{11}+c_{12} & -c_{11} & -c_{12} & 0 & 0 & 0 & 0 & 0 \\
-c_{11} & c_{11}+c_{2}+c_{3} & -c_{2} & -c_{3} & 0 & 0 & 0 & 0 \\
-c_{12} & -c_{2} & c_{12}+c_{2}+c_{4} & 0 & -c_{4} & 0 & 0 & 0 \\
0 & -c_{3} & 0 & c_{3}+c_{5}+c_{57} & -c_{5} & -c_{57} & 0 & 0 \\
0 & 0 & -c_{4} & -c_{5} & c_{4}+c_{5}+c_{68} & 0 & -c_{68} & 0 \\
0 & 0 & 0 & -c_{57} & 0 & c_{6}+c_{57} & 0 & -c_{6} \\
0 & 0 & 0 & 0 & -c_{68} & 0 & c_{7}+c_{68} & -c_{7} \\
0 & 0 & 0 & 0 & 0 & -c_{6} & -c_{7} & c_{6}+c_{7}
\end{array}\right] .}
\end{aligned}
$$

From Figure 5, we can see that the predicted dynamic stiffness is in exact agreement with the direct computation. Hence, the proposed indirect inverse substructuring method demonstrates its validity for identifying the dynamic stiffness at coupling interfaces. It should be noted that the formulation may be sensitive to input random errors. As illustrated 


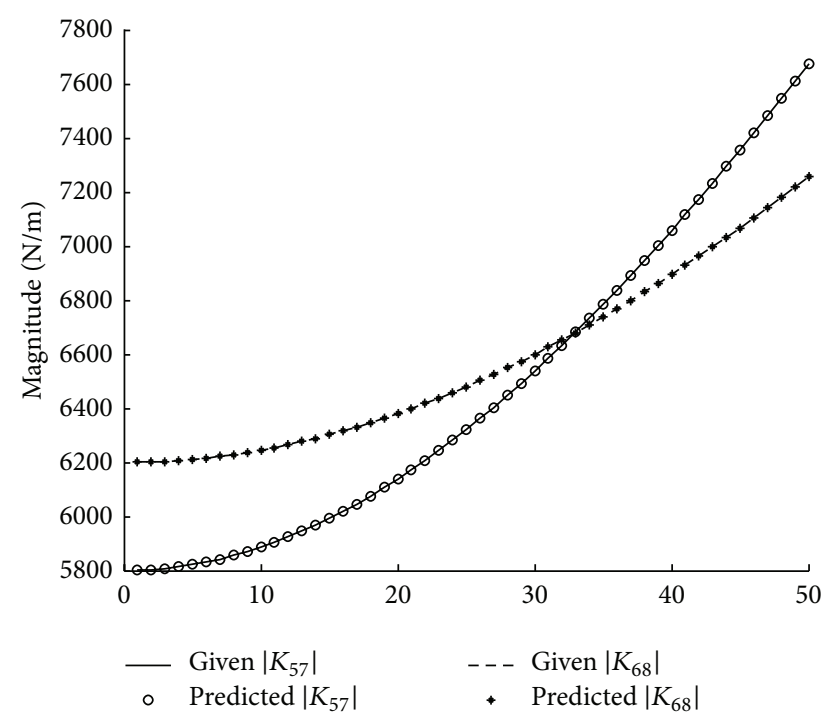

FIGURE 5: Comparison of predicted and exact coupling dynamic stiffness; here, $K_{57}=k_{57}+j \omega c_{57}, K_{68}=k_{68}+j \omega c_{68}$.

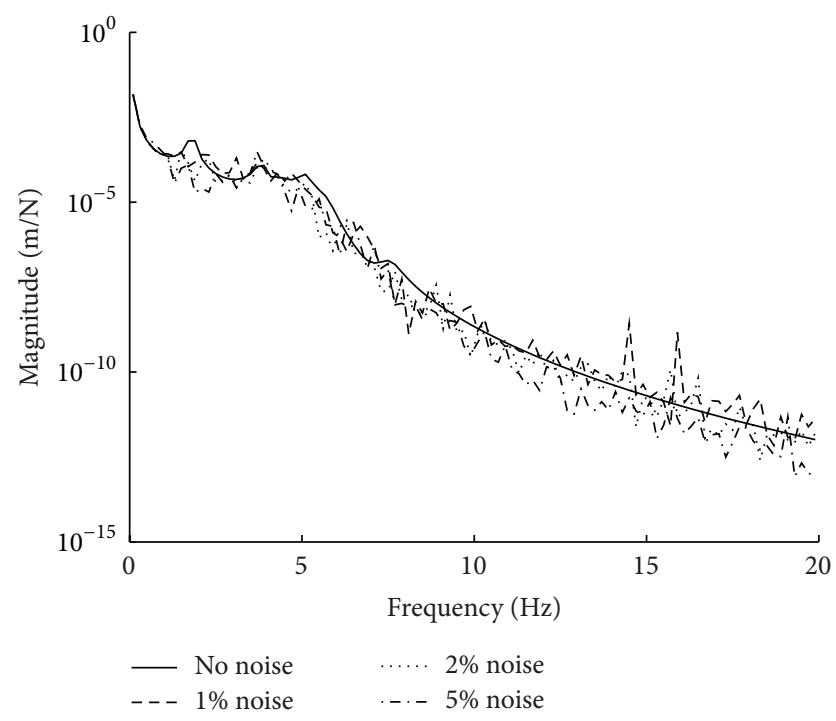

FIGURE 6: Effect of random noise of measured system-level FRFs on the predicted frequency response function from $m_{9}$ to $m_{1}$.

in Figure 6, the predicted system-level frequency response function is affected remarkably by the input random errors of measured system-level frequency response functions, implying that the formulation for prediction of coupling stiffness is extremely sensitive to the experiment errors. A further study on the error propagation as well as the elimination approach will be conducted in the future.

\section{Conclusions}

Three sets of equations for multicomponent coupled product transport system are obtained, including the dynamic equilibrium conditions, the displacement compatibility conditions in addition to the relationship of the system-level
FRFs, and the selected easy-to-monitor component-level FRFs. Then, the closed-form analytical solution to inverse substructuring analysis of multisubstructure coupled product transport system with rigid and flexible coupling is derived. The method developed offers an approach to predict the unknown coupling dynamic stiffness from system-level FRFs and easy-to-monitor component-level FRFs, avoiding the difficulties of vibration excitation and/or measurement in the coupling interface. Then, the proposed method is validated by a lumped mass-spring-damper model, and the coupling dynamic stiffness is compared with the direct computation, showing exact agreement. The method developed offers an approach to predict the unknown coupling dynamic stiffness from system-level FRFs and component-level FRFs which can be easily measured. The suggested method may help to obtain the main controlling factors and contribution from the various structure-borne paths for product transport system, which may certainly facilitate the cushioning packaging design.

\section{Conflict of Interests}

The authors declare that there is no conflict of interests regarding the publication of this paper.

\section{Acknowledgments}

The authors would like to appreciate the financial support of National Natural Science Foundation of China (Grant no. 51205167), Research Fund for the Doctoral Program of Higher Education of China (Grant no. 20120093120014), and Fundamental Research Funds for the Central Universities (Grant no. JUSRP51403A), the project was supported by the Foundation (Grant no. 2013010) of Tianjin Key Laboratory of Pulp \& Paper (Tianjin University of Science \& Technology) and by the Foundation of Jiangsu Key Laboratory of Advanced Food Manufacturing Equipment and Technology (Grant no. FM-201403), and the research was also sponsored by Qing Lan Project of Jiangsu Province (2014).

\section{References}

[1] R. E. Newton, Fragility Assessment Theory and Practice, Monterey Research Laboratory, Monterey, Calif, USA, 1968.

[2] G. J. Burgess, "Product fragility and damage boundary theory," Packaging Technology and Science, vol. 15, no. 10, pp. 5-10, 1988.

[3] Z. Wang, C. Wu, and D. Xi, "Damage boundary of a packaging system under rectangular pulse excitation," Packaging Technology and Science, vol. 11, no. 4, pp. 189-202, 1998.

[4] Z. W. Wang and C. Y. Hu, "Shock spectra and damage boundary curves for nonlinear package cushioning system," Packaging Technology and Science, vol. 12, no. 5, pp. 207-217, 1999.

[5] C. Ge, "Model of accelerated vibration test," Packaging Technology and Science, vol. 13, no. 1, pp. 7-11, 2000.

[6] J. Wang, Z.-W. Wang, L.-X. Lu, Y. Zhu, and Y.-G. Wang, "Three-dimensional shock spectrum of critical component for nonlinear packaging system," Shock and Vibration, vol. 18, no. 3, pp. 437-445, 2011. 
[7] J. Wang, F. Duan, J.-H. Jiang, L.-X. Lu, and Z.-W. Wang, “Dropping damage evaluation for a hyperbolic tangent cushioning system with a critical component," Journal of Vibration and Control, vol. 18, no. 10, pp. 1417-1421, 2012.

[8] F.-D. Lu, W.-M. Tao, and D. Gao, "Virtual mass method for solution of dynamic response of composite cushion packaging system," Packaging Technology and Science, vol. 26, no. S1, pp. 32-42, 2013.

[9] H. Kitazawa, K. Saito, and Y. Ishikawa, "Effect of difference in acceleration and velocity change on product damage due to repetitive shock," Packaging Technology and Science, vol. 27, no. 3, pp. 221-230, 2014.

[10] D. Shires, "On the time compression (test acceleration) of broadband random vibration tests," Packaging Technology and Science, vol. 24, no. 2, pp. 75-87, 2011.

[11] M. A. Sek, V. Rouillard, and A. J. Parker, "A study of nonlinear effects in a cushion-product system on its vibration transmissibility estimates with the reverse multiple input-single output technique," Packaging Technology and Science, vol. 26, no. 3, pp. 125-135, 2013.

[12] Y. Ren and C. F. Beards, "Identification of joint properties of a structure using frf data," Journal of Sound and Vibration, vol. 186, no. 4, pp. 567-587, 1995.

[13] T. Yang, S.-H. Fan, and C.-S. Lin, "Joint stiffness identification using FRF measurements," Computers and Structures, vol. 81, no. 28-29, pp. 2549-2556, 2003.

[14] D. Čelič and M. Boltežar, "Identification of the dynamic properties of joints using frequency-response functions," Journal of Sound and Vibration, vol. 317, no. 1-2, pp. 158-174, 2008.

[15] J. Zhen, T. C. Lim, and G. Lu, "Determination of system vibratory response characteristics applying a spectral-based inverse sub-structuring approach. Part I: analytical formulation," International Journal of Vehicle Noise and Vibration, vol. 1, no. 1-2, pp. 1-30, 2004.

[16] Z.-W. Wang, J. Wang, Y.-B. Zhang, C.-Y. Hu, and Y. Zhu, "Application of the inverse substructure method in the investigation of dynamic characteristics of product transport system," Packaging Technology and Science, vol. 25, no. 6, pp. 351-362, 2012.

[17] J. Wang, L. Lu, and Z. Wang, "Modeling the complex interaction between packaged product and vehicle," Advanced Science Letters, vol. 4, no. 6-7, pp. 2207-2212, 2011.

[18] Z.-W. Wang and J. Wang, "Inverse substructure method of three-substructures coupled system and its application in product-transport-system," Journal of Vibration and Control, vol. 17, no. 6, pp. 943-952, 2011.

[19] J. Wang, X. Hong, Y. Qian, Z.-W. Wang, and L.-X. Lu, "Inverse sub-structuring method for multi-coordinate coupled product transport system," Packaging Technology and Science, vol. 27, no. 5, pp. 385-408, 2014. 

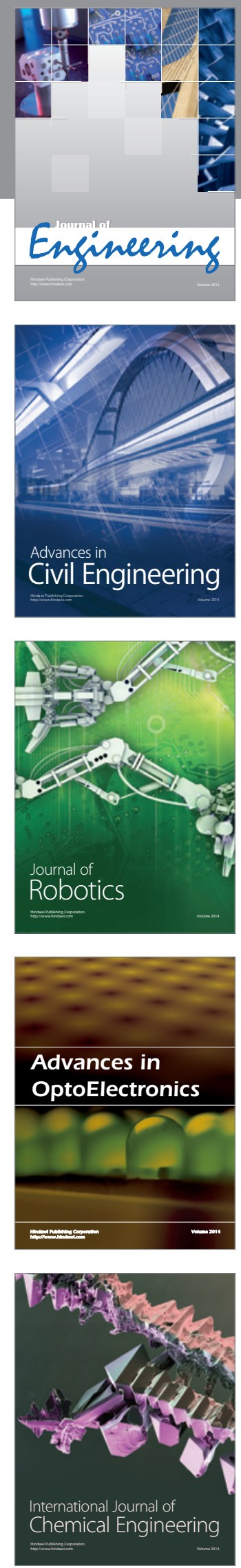

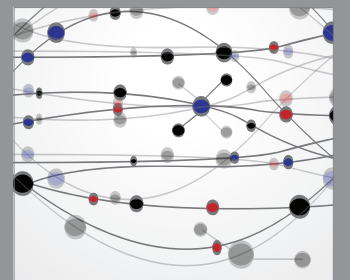

The Scientific World Journal
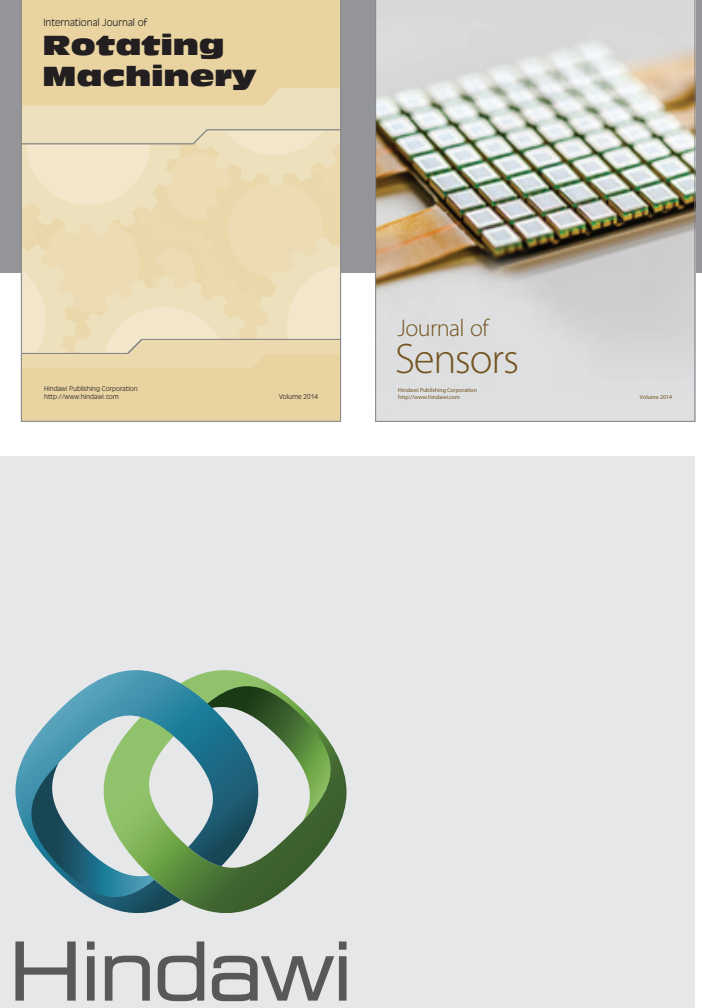

Submit your manuscripts at http://www.hindawi.com
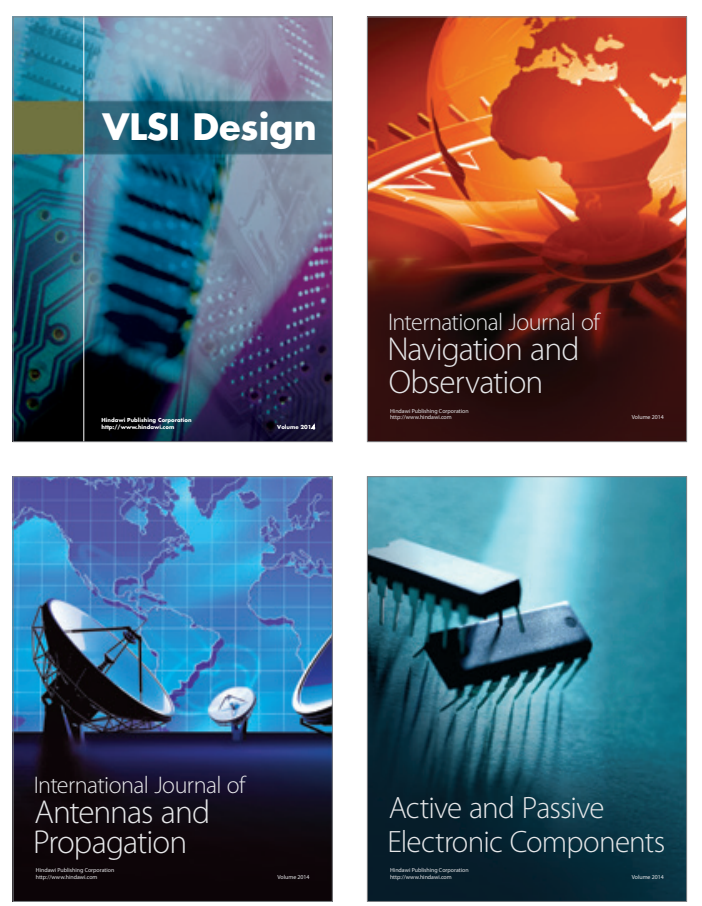
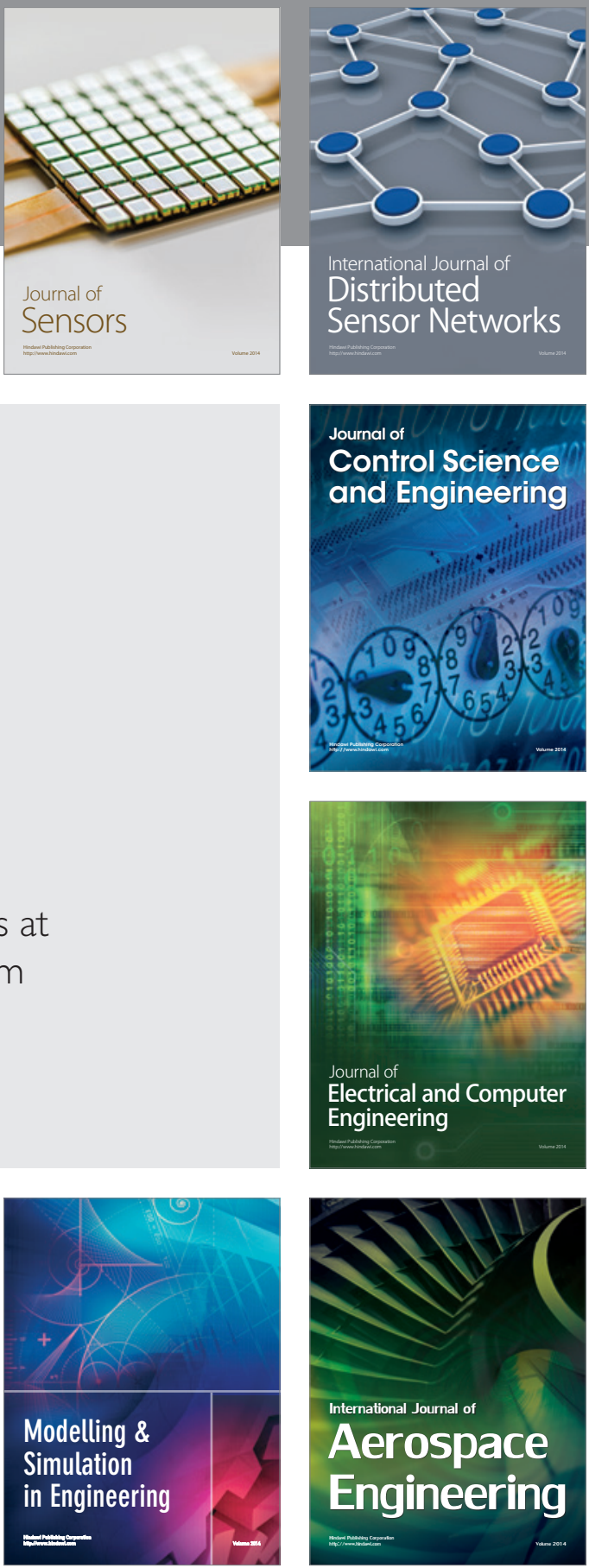

Journal of

Control Science

and Engineering
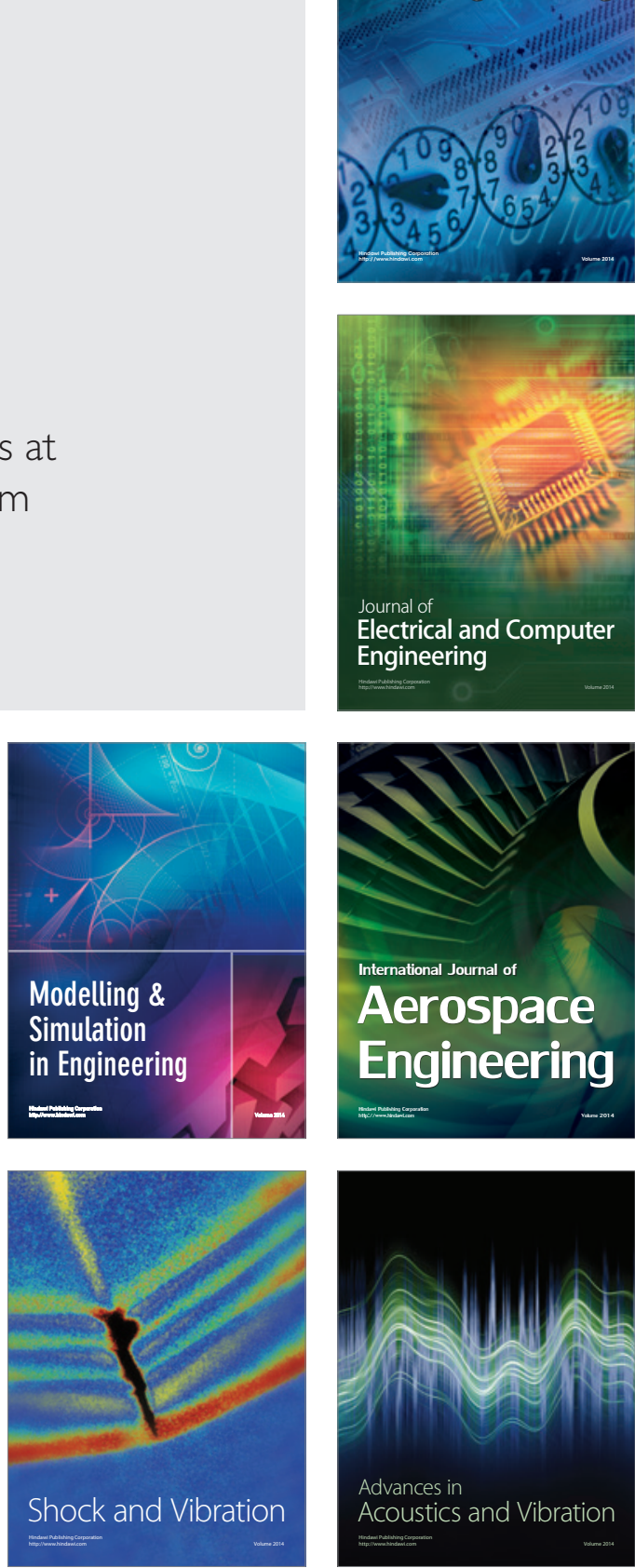